\title{
The impact of sleeping with reduced glycogen stores on immunity and sleep in triathletes
}

\author{
Julien Louis ${ }^{1}$ (D) Laurie-Anne Marquet ${ }^{2,3}$ - Eve Tiollier $^{2}$ - Stéphane Bermon ${ }^{3,4}$. \\ Christophe Hausswirth ${ }^{2} \cdot$ Jeanick Brisswalter $^{3}$
}

Received: 16 May 2016 / Accepted: 26 July 2016 / Published online: 4 August 2016

(C) The Author(s) 2016. This article is published with open access at Springerlink.com

\begin{abstract}
Purpose We investigated the effects of a 3-week dietary periodization on immunity and sleep in triathletes.

Methods 21 triathletes were divided into two groups with different nutritional guidelines during a 3-week endurance training program including nine twice a day sessions with lowered (SL group) or maintained (CON group) glycogen availability during the overnight recovery period. In addition to performance tests, sleep was monitored every night. Systemic and mucosal immune parameters as well as the incidence of URTI were monitored every week of the training/nutrition protocol. Two-ways ANOVA and effect sizes were used to examine differences in dependent variables between groups at each time point.

Results The SL group significantly improved $10 \mathrm{~km}$ running performance ( $-1 \min 13 \mathrm{~s}, P<0.01, d=0.38$ ), whereas no improvement was recorded in the CON group $(-2 \mathrm{~s}, \mathrm{NS})$. No significant changes in white blood cells counts, plasma cortisol and IL-6 were recorded over the protocol in both
\end{abstract}

Communicated by David C. Poole.

Julien Louis

J.B.Louis@ljmu.ac.uk

1 Research Institute for Sport and Exercise Sciences, Liverpool John Moores University, Byrom Street, Liverpool L3 3AF, UK

2 Laboratory of Sport, Expertise and Performance, French National Institute of Sport, Expertise and Performance, Paris, France

3 Laboratory of Human Motricity, Education, Sport and Health, University of Nice Sophia-Antipolis, Nice, France

4 Institut Monégasque de Médecine et Chirurgie du Sport, Monte Carlo, Monaco groups. The vitamin D status decreased in similar proportions between groups, whereas salivary $\operatorname{IgA}$ decreased in the SL group only $(P<0.05, d=0.23)$. The incidence of URTI was not altered in both groups. All participants in both groups went to bed earlier during the training program (SL $-20 \mathrm{~min}, \mathrm{CON}-27 \mathrm{~min}, P<0.05, d=0.28$ ). In the SL group, only sleep efficiency slightly decreased by $1.1 \%$ $(P<0.05, d=0.25)$ and the fragmentation index tended to increase at the end of the protocol $(P=0.06)$.

Conclusion Sleeping and training the next morning regularly with reduced glycogen availability has minimal effects on selected markers of immunity, the incidence of URTI and sleeping patterns in trained athletes.

Keywords Carbohydrate - Dietary manipulation - Immune response $\cdot$ Sleep pattern $\cdot$ Endurance training $\cdot$ Upper respiratory tract infection

\begin{tabular}{ll}
\multicolumn{2}{l}{ Abbreviations } \\
ANOVA & Analysis of variance \\
CHO & Carbohydrate \\
CON group & Control group \\
HIT & High intensity training \\
LIT & Low intensity training \\
MAP & Maximal aerobic power \\
RER & Rate of exchange ratio \\
sIgA & Salivary immunoglobulin-A \\
sIL-6 & Salivary interleukin-6 \\
SL group & Sleep low group \\
URTI & Upper respiratory tract infection \\
$V C O_{2}$ & Carbon dioxide uptake \\
$V \mathrm{O}_{2}$ & Oxygen uptake \\
$V \mathrm{O}_{2 m a x}$ & Maximal oxygen uptake \\
$\mathrm{WURSS}_{2} 21$ & Wisconsin upper respirtory symptor
\end{tabular}

WURSS-21 Wisconsin upper respiratory symptom survey-21 


\section{Introduction}

Training strategies with low glycogen availability are increasingly used by endurance athletes in an attempt to improve performance thus also increasing scientific interest for exercise physiologists (Bartlett et al. 2015). The main expected effect of "training low" is to enhance training stress and thus physiological adaptations related to endurance performance. Several studies have reported an enhanced expression of a number of genes related to the stress response, substrate utilization, and mitochondrial biogenesis in athletes experiencing training low strategies compared to training with normal glycogen availability (Bartlett et al. 2015; Impey et al. 2016). Several "training low" strategies are used by endurance athletes such as training in a fasted state (i.e., 6-10 $\mathrm{h}$ after the last meal), training twice per day (where the second session is performed with reduced glycogen stores), or restricting carbohydrates (CHO) intake during the recovery period after exercise (Hansen et al. 2005; Morton et al. 2009; Van Proeyen et al. 2011; Yeo et al. 2008). Recently, the "sleeping low" strategy has been introduced, consisting of training in the evening followed by overnight fast and performing a subsequent training session in the morning, to accentuate the glycogen deprivation without altering exercise intensity (Lane et al. 2015; Marquet et al. 2016). Taken together, results from the literature suggest that the most stressful training situation may provide the greatest physiological adaptation. However, it is well known that increasing training stress could influence immune function and increase the risk of illness and/or injury limiting improvements in performance (Gleeson 2007).

In endurance sports, training load, nutrient intake and sleep are key factors modulating immune function either positively or negatively (Gleeson 2007; Nieman 2000). Regular physical activity, as well as balanced diet and enough sleep are associated with improved immune function (Fullagar et al. 2015; Nieman 1998). On the contrary, prolonged or strenuous exercise, energetic deficit and a lack of sleep, decrease immune function and increase susceptibility to infections and pathologies. Several studies have reported an increased prevalence of upper respiratory tract infections (URTI) during overload training programs and after endurance events such as marathon races (Bermon 2007; Pyne et al. 1995). For example, Nieman et al. (1990) recorded that $13 \%$ of participants reported URTI during the week following the Los Angeles Marathon race and $40 \%$ reported at least one episode of URTI during the 2 months prior to the race. Many components of the immune system exhibit changes after endurance exercise (Hoffman-Goetz and Pedersen 1994). This alteration in immune function reaches its summit within a window of 3-72 $\mathrm{h}$ after exercise reflecting the physiological stress the endurance athlete's body is experiencing (Gleeson 2007). During this "open window" phenomenon, the body's ability to fight infections is dramatically lowered, typically associated with changes in leukocyte counts and production of interleukins and immunoglobulins (Gleeson 2007). A reduction in carbohydrate availability during exercise may accentuate the alteration of immune function. Several authors have shown that very low $\mathrm{CHO}$ diets $(<10 \%$ of energy intake from $\mathrm{CHO}$ ) induce an alteration of immune response compared to normal or high $\mathrm{CHO}$ diets (Bishop et al. 2001a; Mitchell et al. 1998). Although normal or high $\mathrm{CHO}$ availability during endurance exercise is effective in attenuating some immune perturbations, there is still no evidence that these beneficial effects on immune parameters are clinically relevant.

In addition to adapted dietary intake, optimized sleep patterns constitute a prerequisite for a good assimilation of training load (Myllymaki et al. 2011; Samuels 2008). Sufficient sleep participates greatly in the maintenance of the body homeostasis necessary to endure training sessions apart from injuries and infections. More than the sleep duration, the rhythmic cycle of sleep and wakefulness have important implications on the regulation of several hormones involved in the management of fatigue and immune function (Shepard and Shek 1996). Although regular exercise has been shown to improve sleep quality, the latter can be affected during intense training periods (Hausswirth et al. 2014; Leeder et al. 2012; Taylor et al. 1997). A significant reduction in sleep duration $(-6 \%)$, sleep efficiency $(-2 \%)$ and time in bed $(-3 \%)$, and an increase in wakefulness after sleep onset $(+3 \%)$, were recorded in classical ballet dancers at the end of 67 days of high physical training before a premiere performance (Fietze et al. 2009). Similarly, Hausswirth et al. (2014) reported a significant decrease in sleep duration $(-7.9 \%)$, sleep efficiency $(-1.6 \%)$ and immobile time $(-7.6 \%)$ after 3 weeks of overload training in triathletes. These sleep disturbances were associated with a higher prevalence of URTI $(+67 \%)$ compared with a control group without increase in training load. Additionally, some studies suggest that nutritional feedings may alter the sleep quality (Halson 2014). Consuming $\mathrm{CHO}$ in a solid meal could reduce sleep latency, while diets rich in protein may result in enhanced sleep quality by limiting wake episodes (Afaghi et al. 2008; Lindseth et al. 2013). There is limited research in this area and additional studies are necessary to evaluate the influence of habitual diet on sleep patterns, and the combined effects of strenuous exercise and nutritional manipulation.

The aim of this study was to assess whether the sleep low strategy, consisting of sleeping with reduced glycogen availability might alter sleep patterns (i.e., sleep quantity and quality) and immune response in trained triathletes. Using the same experimental protocol, this study is a complement to that 
Fig. 1 Schematic representation of the experimental protocol

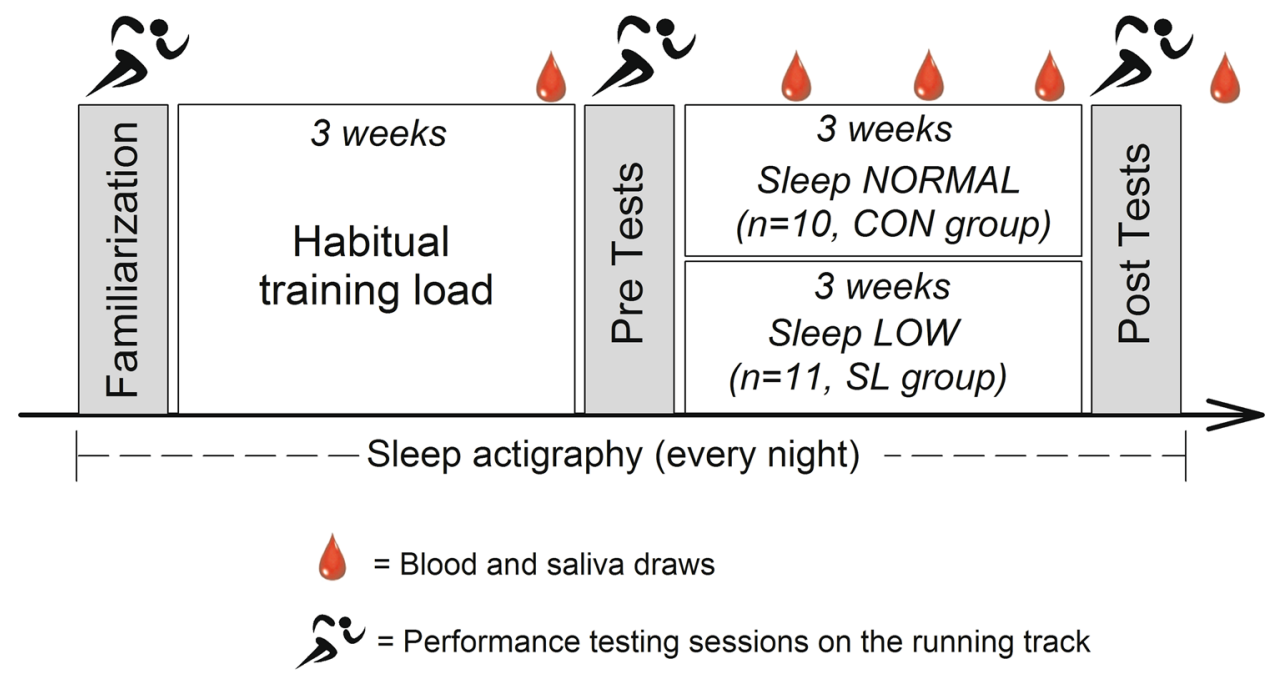

presented by Marquet et al. (2016) giving us the opportunity to provide new insight on the potential side effects of the promising sleep low strategy. In light of past literature, we hypothesized that when sleeping low, athletes would experience an alteration of immune function accompanied with a higher prevalence of URTI, as well as disturbances in sleep patterns.

\section{Methods}

\section{Participants}

Twenty-one trained male triathletes volunteered to participate in this study. Subjects could be included if they were currently healthy, aged 18-40 years, had been involved in endurance training and competition for at least 2 years, and trained at least $10 \mathrm{~h}$ per week including several moderate to high intensity training sessions per week. Their mean ( \pm SD) age, height, body mass, maximal oxygen uptake, and maximal aerobic power were $31 \pm 4.7$ years, $1.79 \pm 0.05 \mathrm{~m}$, $71.6 \pm 4.5 \mathrm{~kg}, 4.2 \pm 0.4 \mathrm{~L} \cdot \mathrm{min}^{-1}, 336.6 \pm 31.4 \mathrm{~W}$. Before the experiment a cardiologist examined all the participants to check they did not present contraindications to physical exercise and to ensure normal electrocardiograph patterns. All subjects were free of URTI symptoms for at least 2 weeks and had not taken any medication in the 4 weeks prior to the study. The experimental design of the study was approved by the local Ethics Committee (Paris IDF VI, France) and was carried out according to the Declaration of Helsinki. After comprehensive verbal and written explanations of the study, all subjects gave their written informed consent to participate.

\section{Study design}

An overview of the study design is shown in Fig. 1. This study was conducted to analyze the potential effects of chronic reduced glycogen availability during and between endurance training sessions on sleep pattern, selected immune parameters and the incidence of URTI. 21 trained triathletes were randomly assigned to two different groups undertaking the same endurance training program for three consecutive weeks with different nutritional guidelines. Although one group was instructed to reduce its $\mathrm{CHO}$ intake during and between training sessions according to a sleep low design (named Sleep Low group, SL group), the other group maintained regular $\mathrm{CHO}$ intake over the day (named Control group, CON group) so that the SL group slept with low glycogen availability while the other group slept with normal $\mathrm{CHO}$ availability. In addition to performance tests in running, sleep patterns, immune parameters and the incidence of URTI were regularly assessed before, during and after the training program.

\section{Training protocol}

After preliminary testing sessions, all participants were involved in a 6-week training program, which was divided into two distinct phases interspersed with testing sessions. The first phase (I) consisted of 3 weeks during which the participants completed their usual training regime $(10-15 \mathrm{~h}$ per week at various intensities). This first training period was organized to ensure that all participants were regularly involved in an endurance training and in a similar training status before the beginning of the study. The second phase (II) consisted of 3 weeks during which all participants completed the same standardized training program with different nutritional guidelines. The training program consisted of six training sessions over four consecutive days, including high intensity training (HIT) sessions in the afternoon (after $5 \mathrm{pm}$ ) and low intensity training (LIT) sessions in the next morning (before $10 \mathrm{am}$ ). The training intensity was individually set according to the individual maximal 

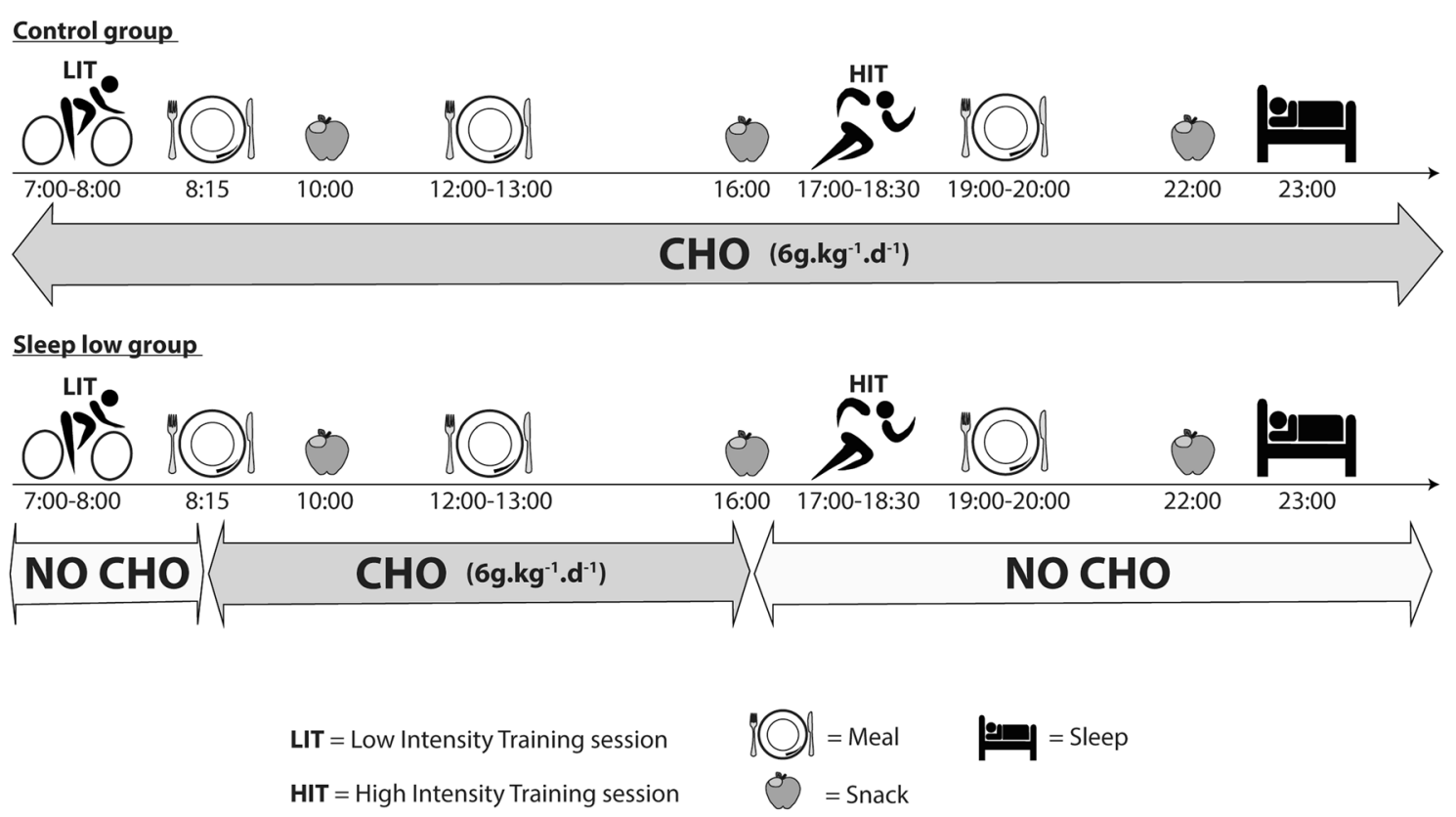

Fig. 2 Daily CHO periodization for SL and CON groups during training days in phase II. CHO intake was distributed at every meal, snack and training sessions in the CON group, whereas it was concentrated only from breakfast ( 8:15) to afternoon snack $(\sim 16: 00)$ in the SL group

aerobic power (MAP). All LIT sessions consisted of $60 \mathrm{~min}$ cycling at $65 \%$ MAP, while HIT sessions consisted alternatively of $8 \times 5$ min cycling at $85 \%$ MAP and $6 \times 5$ min running at individual $10 \mathrm{~km}$ intensity with $1 \mathrm{~min}$ recovery between sets. One LIT session per day was prescribed for the other days of the week to maintain a $10-15 \mathrm{~h}$ training volume. All participants performed all the training sessions in their own training structure and were monitored (activity, duration, intensity, rate of perceived exertion) and controlled by heart rate recordings.

\section{Nutritional protocol}

Before phase II, participants were randomly assigned to either the CON group $(n=10)$ or the SL group $(n=11)$ and had to follow different nutritional guidelines according to their group. In the SL group, no CHO intake was allowed for all HIT and LIT sessions. The dinner was also CHOfree and the LIT sessions were performed after an overnight fast so that they trained with low glycogen availability. On the contrary, the glycogen availability was regularly maintained in the CON group by consuming a sports drink (4.5\% CHO, Gatorade Performance Series ${ }^{\circledR}$, Pepsico, USA) during training sessions and $\mathrm{CHO}$ at every meal. Finally all groups ingested the same amount of $\mathrm{CHO}$ per day $\left(\sim 6 \mathrm{~g} \cdot \mathrm{kg}^{-1}\right.$.day $\left.{ }^{-1}\right)$ but allocated differently over each day. A typical daily $\mathrm{CHO}$ periodization for both groups during training days in phase II is depicted in Fig. 2. All participants received standardized dietary recommendation according to their membership group and their body weight. To avoid muscle catabolism, a high protein sugarfree drink (High Protein 15 g, 20 mL, UHS Bruno, France) was prescribed just before going to bed.

The participants had to fill in a food diary during the last week in phase I and phase II. They were instructed to fill in it the most detailed as possible (weighed food, pictures of dishes, details of the use of cooking fat, type and quantity of oil used for dressing...). The diaries were analyzed by the same scientist with the software Nutrilog 2.31 (Nutrilog SAS, France).

\section{Measurements}

\section{$\dot{V} \mathrm{O}_{2 \max }$ and performance tests}

For their first visit to the lab, subjects underwent an incremental cycling test at a self-selected cadence on an electronically braked cycle ergometer (Excalibur Sport, Lode ${ }^{\circledR}$, Groningen, The Netherlands). The test consisted of a warm-up lasting $6 \mathrm{~min}$ at $100 \mathrm{~W}$ followed by an incremental period in which power output was increased by $25 \mathrm{~W}$ every 2 min until volitional exhaustion. The test was performed until exhaustion to assess maximal oxygen uptake $\left(\dot{V} \mathrm{O}_{2 \max }\right)$ and maximal aerobic power (MAP). During the test, oxygen uptake $\left(\dot{V} \mathrm{O}_{2}\right)$, carbon dioxide uptake $\left(\dot{V} \mathrm{CO}_{2}\right)$, minute ventilation $(\dot{V} E)$ and respiratory exchanges ratio (RER) were continuously recorded and monitored as breath by breath values (Quark, Cosmed ${ }^{\circledR}$, Rome, Italy). 
The gas and flow analyzers were calibrated prior to each test using ambient air, known-concentration gas and a $3 \mathrm{~L}$ syringe. $\dot{V} \mathrm{O}_{2}$ max was determined by the highest $30 \mathrm{~s}$ average value. MAP $(W)$ was calculated as MAP $=W$ completed $+25 \times(t / 120)$ where $W$ is the last completed workload and $t$ is the number of seconds in the last workload completed or not.

The performance test was the same presented in the recent study published by Marquet et al. (2016). Briefly it was organized to assess the potential changes in endurance performance in ecological condition. It was planned during the first week as a familiarization trial, and immediately before and after the Phase II. This test was designed to simulate the end of a triathlon race. The test started by 40 min cycling at $70 \% \mathrm{MAP}$ at a self-selected cadence, immediately followed by a $10 \mathrm{~km}$ simulated running race. To allow the subject to drink during the exercise, two short active rest periods (30 s at $100 \mathrm{~W}$ at minutes 15 and 30) were organized, during which a water bottle was given to the subject. Immediately after the cycling exercise, the subjects quickly moved to the running track (340 $\mathrm{m}$ indoor) to start a $10 \mathrm{~km}$ test. During this test, subjects did not wear any apparatus and could drink a CHO-rich drink $(4.5 \mathrm{~g}$ $\mathrm{CHO}$ per liter, Gatorade Performance Series-Endurance Formula) whenever they wanted. The bottle was placed on a table positioned on the running track. The bottle was regularly replaced on the table after each drink and weighed before and after the running test to evaluate the fluid intake. No significant difference was observed for the quantity of $\mathrm{CHO}$ ingested between performance tests $(P=0.62)$ and between groups (respectively for SL group; PRE vs. POST $14.7 \pm 7.21$ vs. $15.3 \pm 6.43 \mathrm{~g} ; P=0.47$ and for CON group PRE vs. POST $18.0 \pm 15.5$ vs. $15.1 \pm 11.3 \mathrm{~g}$; $P=0.50)$ The time lap was continuously recorded by an experimenter positioned on the track.

\section{Blood collection and analyses}

To avoid interassay variation, all blood samples were analyzed in a single batch at the end of the study. In five occasions (before and after the phase II, and before the last training session each week of the phase II, day 4, 11 and 18) in a fasted state, blood samples were collected from a superficial forearm vein using standard venipuncture techniques. $33 \mathrm{~mL}$ of blood was directly collected into EDTA tubes $(2$ EDTA tubes $=6 \mathrm{~mL}$ and 1 EDTA tube $=3 \mathrm{~mL}$ ) for each sample (Greiner Bio-one; Frickenhausen, Germany).

Blood samples were immediately centrifuged at $4000 \mathrm{rev} \mathrm{min}^{-1}$ for $10 \mathrm{~min}$ at $+4{ }^{\circ} \mathrm{C}$ to separate plasma from red blood cells. The obtained plasma sample was then stored in multiple aliquots (Eppendorf type, $1500 \mu \mathrm{L}$ per sample) at $-80{ }^{\circ} \mathrm{C}$ until analysis. From these samples, cortisol and vitamin D concentrations were determined in plasma with commercially available high sensitivity ELISA kits (R\&D Systems, Minneapolis, MN, USA). The assay for [cortisol]b had an intraassay CV of 9.2-6.3\% over a concentration range of $1.3-6.5 \mu \mathrm{g} \mathrm{L}^{-1}$ and an interassay $\mathrm{CV}$ of $21.2-10.4 \%$ over $1.1-5.5 \mu \mathrm{g} \mathrm{L}^{-1}$. The assay for [vitamin D]b had an intraassay CV of 5.7-6.2\% over a concentration range of $33-180 \mathrm{ng} \mathrm{mL}^{-1}$ and an interassay $\mathrm{CV}$ of 5.1-7.4 \% over 52.9-164 ng mL ${ }^{-1}$. All blood samples were analyzed in duplicate at respective wavelength on a spectrophotometer Dynex MRXe (Magellan Biosciences, Chelmsford, MA, USA). Blood from $3 \mathrm{~mL}$ tubes was analyzed for leukocyte count using an automated cell counter (Cell-DynH RubyTM, Abbott, IL, USA) and standard laboratory procedures.

\section{Saliva collection and analyses}

On five occasions (before and after the phase II, and before the last training session each week of the phase II, day 4, 11 and 18) in a fasted state, athletes provided saliva samples. The samples were collected in multiple sterile aliquots (Eppendorf type, $3000 \mu \mathrm{L}$ per sample) over a timed 5-min period and stored at $-80{ }^{\circ} \mathrm{C}$ until assay. After thawing, saliva samples were centrifuged at $4000 \mathrm{rev} \mathrm{min}^{-1}$ for $10 \mathrm{~min}$ at $+4{ }^{\circ} \mathrm{C}$. The samples were analyzed for salivary immunoglobulin-A (sIgA) and interleukin-6 (IL-6) in accordance with the manufacturer's recommendations (EIA kits, Salimetrics@, State College, PA, USA). The assay for $[\operatorname{IgA}] \mathrm{s}$ had an intraassay $\mathrm{CV}$ of $4.5-6.9 \%$ over a concentration range of 91.1-805.4 $\mu \mathrm{g} \mathrm{mL}-1$ and an interassay $\mathrm{CV}$ of 8.9-8.6\% over $25.3-204.1 \mu \mathrm{g} \mathrm{mL}{ }^{-1}$. The assay for [IL-6]s had an intraassay $\mathrm{CV}$ of 3-10\% over a concentration range of 4-323 pg mL $\mathrm{mL}^{-1}$ and an interassay $\mathrm{CV}$ of $8-6 \%$ over 9-342 $\mu \mathrm{g} \mathrm{mL}^{-1}$.

\section{Illness symptoms}

The occurrence of upper tract respiratory infections (URTI) was evaluated using the Wisconsin Upper Respiratory Symptom Survey-21 (WURSS-21) (Barrett et al. 2009). The WURSS-21 includes one global severity question, ten symptom-based questions, nine functional impairment or quality-of-life questions, and one global change question. The severity of each reported symptom was rated on a seven-point scale: 1 (very mild), 3 (mild), 5 (moderate), and 7 (severe). An overall symptom score was calculated by adding the severity scores from all items except the first and the last as they have categorically different reference domains. The questionnaire was performed every day on the 3 weeks of phase II, and was administered at the same period of the day in a quiet place. All athletes had prior knowledge about the completion of the questionnaire. For 
comparison between weeks, the average of all answered questionnaires at each stage was used. Higher scores indicate more severe symptoms (the theoretical maximum score being 133) whilst a score of 0 indicates the complete absence of symptoms.

\section{Sleep monitoring}

During both phases I and II, subjects' sleep patterns (i.e., sleep quantity and quality) were monitored continuously using an Actiwatch worn on the non-dominant wrist (Cambridge Neurotechnology Ltd., UK) with the epoch length set to $1 \mathrm{~min}$. Athletes were monitored in their home environment every day during phase I ( 21 days), and during phase II (21 days). Mean behavioral activity over the entire recording period was automatically calculated using the Sleepwatch software (Actiwatch activity and sleep analysis version 5.28, Cambridge Neurotechnology, Ltd.). Wristwatch actigraphy is a non-intrusive tool used to estimate sleep efficiency, which has been validated for reliability (Sadeh 2011). When compared with polysomnography, results show an accuracy of up to $80 \%$ in sleep disordered patients for total sleep time and sleep efficiency (Kushida et al. 2001).

Sleep-wake scoring can be reliably obtained only with additional information provided by manually completed sleep logs (Fietze et al. 2009). All participants were, therefore, requested to complete daily sleep diaries, indicating the times of going to bed, falling asleep, waking up, and leaving the bed. In addition, participants were asked to mark the time of switching off the light to sleep and wakeup time by pressing the button on the face of the Actiwatch.

Individual nights of sleep were analyzed for the following range of variables:

- time in bed (h): the total amount of time spent in bed between bedtime and get-up time;

- bedtime (hh:mm): the self-reported clock time at which a participant went to bed to attempt to sleep;

- get-up time (hh:mm): the self-reported clock time at which a participant got out of bed;

- sleep latency (min): the amount of time between bedtime and sleep start;

- actual sleep time (hh:mm): assumed sleep time as determined by the algorithm, taking into account immobile time;

- sleep efficiency (\%): actual sleep time expressed as a percentage of time in bed;

- fragmentation index: a measure of restlessness during sleep, using the percentage of epochs where activity is $>0$;

- immobile time (min): the actual time spent immobile in bed.
To quantify how the training weeks affected the perceived sleep quality, the participants reported their perceived feelings on a seven-point scale, going from very, very good $(=1)$ to very, very poor $(=7)$ after waking up each morning (Hooper et al. 1995).

\section{Statistical analysis}

All statistical analyses were conducted using the software Statistica 6.1 (StatSoft). All data are expressed as mean \pm SD. Normality of data was tested using a Shapiro-Wilk test. Values at baseline for age, body composition, and experience in endurance sport, MAP, $V \mathrm{O}_{2 \max }$ and dietary habits were compared between groups (i.e., sleep low, SL and sleep normal, CON) using a one-way ANOVA. Two-ways (group $\times$ time) ANOVA were used to examine differences in dependent variables (i.e., sleep characteristics, perceived sleep, illness symptoms, blood and saliva markers of immune response) between groups means at each time point of the protocol. When a significant main effect was found, pairwise comparisons were conducted using Newman-Keuls post hoc analysis. Effect sizes were also calculated using partial eta squared $\left(\eta_{\mathrm{p}}^{2}\right)$ values. Values of $0.1,0.3$ and over 0.5 were, respectively, considered as small, medium and large effects. For all tests, the significance level was set at $P<0.05$.

\section{Results}

Data presented in this article derived from the same experimental protocol presented by Marquet et al. (2016) and thus are complementary to those already presented by Marquet et al. (2016).

\section{Effects on chronometric performance on the $10 \mathrm{~km}$ running race}

A significant enhancement of the chronometric performance on the simulated $10 \mathrm{~km}$ running race was recorded at the end of the training program for all participants of the SL group, whereas no difference was recorded in the CON group (for more details about performance tests, refer to Marquet et al. 2016).

\section{Effects on dietary patterns over the experimental protocol}

The macronutrient intake significantly changed between phase II and phase I in similar proportions between SL and CON groups, mainly with an increase in carbohydrate and protein intake between phase I and II without significant 
changes in energy intake (for more details about the macronutrient intake, refer to Marquet et al. 2016).

As depicted in Table 1(A) and (B), the micronutrient intake (vitamin $\mathrm{A}, \mathrm{B}_{1}, \mathrm{~B}_{2}, \mathrm{~B}_{3}, \mathrm{~B}_{6}, \mathrm{~B}_{9}, \mathrm{~B}_{12}, \mathrm{C}, \mathrm{D}, \mathrm{E}$ and magnesium, calcium, phosphorus, potassium, sodium, iron, zinc, copper, manganese, selenium) was not significantly altered between phase II and phase I and no significant difference was recorded between SL and CON groups.

\section{Effects on blood and saliva immune and inflammatory variables}

There were no significant differences in circulating numbers of leukocytes, neutrophils, monocytes, eosinophils, basophils or lymphocytes between groups and between the different phases of the protocol (Table 2). Plasma cortisol levels were not modified throughout the protocol and were not different between groups (Table 3). No significant change was recorded in salivary IL-6 concentration (Table 3). Salivary IgA decreased over the experimental protocol only in the SL group (Table 3; from 391.8 to $245.1 \mu \mathrm{g} \mathrm{L}^{-1}, P<0.05, d=0.23$ from PRE to POST intervention). The vitamin $\mathrm{D}$ status decreased along the protocol in similar proportions between groups (Fig. 3; from $29.6 \pm 7.4$ to $27.8 \pm 5.9 \mathrm{ng} \mathrm{mL}^{-1}$ and from $22.4 \pm 10.6$ to $19.4 \pm 8.1 \mathrm{ng} \mathrm{mL}^{-1}, P<0.05, d=0.18$ between PRE and POST intervention, in SL and CON groups, respectively).

\section{Effects on the incidence of URTI}

The distribution of the daily WURSS-21 scores for both groups is depicted in Fig. 4. The 3 weeks of sleep low program did not modify significantly the WURSS- 21 scores, in comparison with the CON group. The SL group's average score (4.5) was very low and not found to differ to a significant extent compared to the CON group (7.7). The maximum scores recorded were 59 for one subject (1 day in the second week) of the SL group and 39 for one subject in the CON group (1 day in the first week).

\section{Effects on perceived sleep quality and sleep actigraphy}

A summary of variables related to sleep quality and quantity is presented in Table 4. There was no significant timegroup interaction in perceived sleep quality, get-up time, sleep latency, fragmentation index, and number of immobile minutes between phases I and II. In both groups, all subjects went to bed earlier (on average -20 and $-27 \mathrm{~min}$ in the SL and CON groups, respectively, $P<0.05$, $d=0.28$ ) in the phase II than phase I and woke-up at the same time. As such, time in bed significantly increased in similar proportions in both groups (on average +13 and $+15 \mathrm{~min}$ in the SL and CON groups, respectively, $P<0.05$, $d=0.22$ ). However, as depicted in Fig. 5, the actual sleep duration (i.e., the time asleep from sleep start to sleep end, less awakening episodes) was not significantly modified between phase I and II in both groups. Sleep efficiency slightly decreased only in the SL group (Fig. 6; $P<0.05$, $d=0.25)$ and the fragmentation index tended to increase only in the SL group (Fig. 7; $P=0.06$ ).

\section{Discussion}

A growing number of studies support the interest of performing some training sessions with a low glycogen availability to enhance the adaptation to training and endurance performance. In a recently published work from our team (Marquet et al. 2016), we showed a significant improvement in endurance performance and running efficiency following a 3-week training program including the sleep low strategy, i.e., delaying the replenishment of glycogen stores over night and training fasted the next morning. However, reducing the energy availability around training sessions is also well known to increase the risk of mal adaptation to training, including immunodepression and an increased incidence of URTI. This is the reason why we also investigated the effect of the sleep low training program presented by Marquet et al. (2016) on selected immune parameters, the prevalence of URTI and sleep disturbances in endurance athletes. One of the main complementary findings presented in this article is that the enhancement of performance previously described after the SL protocol (Marquet et al. 2016) was accompanied by a slight decrease in sleep efficiency and slight alterations of some indicators of immune function.

\section{Effects on immune parameters and the incidence or URTI}

The effects of exercise on immune function are well documented in the literature. Taken together, results indicate that exercise can have either positive or negative impact on immunity, depending on the nature, intensity and duration of exercise, as well as athlete fitness (Nieman 2000). Regular moderate exercise may enhance immunity and lower the risk or URTI by $20-45 \%$ compared with a sedentary lifestyle (Matthews et al. 2002; Nieman et al. 2011). On the other hand, heavy exercise or periods of chronic exercise may impair immune function and raise the risk of URTI by decreasing resting levels of saliva secretory immunoglobulin-A (sIgA), leukocytes and neutrophil function (Fahlman and Engels 2005; Gleeson 2007; Gleeson et al. 1999). In addition, inadequate dietary feedings such as deficiency in specific macro- and/or micronutrients or negative energy balance, may contribute to impaired immunity and increase 
Table 1 (A) Vitamin and (B) minerals intake for SL and CON groups in phase I and phase II

\begin{tabular}{|c|c|c|}
\hline & Phase I & Phase II \\
\hline \multicolumn{3}{|l|}{ (A) } \\
\hline \multicolumn{3}{|c|}{ Vit A $(\mu \mathrm{g})$} \\
\hline SL & $428.5 \pm 238.8$ & $281.8 \pm 115.9$ \\
\hline $\mathrm{CON}$ & $367.9 \pm 134.0$ & $350.6 \pm 101.8$ \\
\hline \multicolumn{3}{|c|}{ Vit $B_{1}(\mathrm{mg})$} \\
\hline SL & $1.3 \pm 0.5$ & $1.2 \pm 0.6$ \\
\hline $\mathrm{CON}$ & $1.4 \pm 0.5$ & $2.0 \pm 0.7$ \\
\hline \multicolumn{3}{|c|}{ Vit $B_{2}(\mathrm{mg})$} \\
\hline SL & $1.9 \pm 0.6$ & $1.6 \pm 0.6$ \\
\hline $\mathrm{CON}$ & $2.0 \pm 0.7$ & $1.8 \pm 0.4$ \\
\hline \multicolumn{3}{|c|}{ Vit $B_{3}(m g)$} \\
\hline SL & $21.2 \pm 6.2$ & $25.4 \pm 11.0$ \\
\hline $\mathrm{CON}$ & $25.3 \pm 8.1$ & $25.5 \pm 8.9$ \\
\hline \multicolumn{3}{|c|}{ Vit $B_{6}(\mathrm{mg})$} \\
\hline SL & $1.8 \pm 0.4$ & $2.0 \pm 0.7$ \\
\hline $\mathrm{CON}$ & $2.1 \pm 0.9$ & $1.9 \pm 0.5$ \\
\hline \multicolumn{3}{|c|}{ Vit $B_{9}(\mu \mathrm{g})$} \\
\hline SL & $267.2 \pm 86.8$ & $293.9 \pm 117.6$ \\
\hline $\mathrm{CON}$ & $309.4 \pm 155.3$ & $296.1 \pm 81.4$ \\
\hline \multicolumn{3}{|c|}{ Vit $B_{12}(\mu \mathrm{g})$} \\
\hline SL & $4.4 \pm 2.0$ & $4.4 \pm 1.5$ \\
\hline $\mathrm{CON}$ & $4.4 \pm 1.7$ & $4.4 \pm 0.4$ \\
\hline \multicolumn{3}{|c|}{ Vit C (mg) } \\
\hline SL & $109.2 \pm 50.4$ & $130.7 \pm 50.4$ \\
\hline $\mathrm{CON}$ & $141.0 \pm 113.2$ & $132.3 \pm 37.9$ \\
\hline \multicolumn{3}{|c|}{ Vit D ( $\mu \mathrm{g})$} \\
\hline SL & $9.3 \pm 2.2$ & $9.3 \pm 0.7$ \\
\hline $\mathrm{CON}$ & $9.4 \pm 0.9$ & $9.2 \pm 1.1$ \\
\hline \multicolumn{3}{|c|}{ Vit E (mg) } \\
\hline SL & $8.4 \pm 3.2$ & $7.4 \pm 3.1$ \\
\hline $\mathrm{CON}$ & $7.3 \pm 1.4$ & $5.6 \pm 0.9$ \\
\hline \multicolumn{3}{|l|}{ (B) } \\
\hline \multicolumn{3}{|c|}{ Magnesium (mg) } \\
\hline SL & $350.6 \pm 110.5$ & $383.7 \pm 143.3$ \\
\hline $\mathrm{CON}$ & $406.7 \pm 131.0$ & $416.3 \pm 171.1$ \\
\hline \multicolumn{3}{|c|}{ Calcium (mg) } \\
\hline SL & $788.2 \pm 315.8$ & $844.5 \pm 229.8$ \\
\hline $\mathrm{CON}$ & $961.0 \pm 286.2$ & $950.4 \pm 215.6$ \\
\hline \multicolumn{3}{|c|}{ Phosphorus (mg) } \\
\hline SL & $1242.9 \pm 370.3$ & $1485.8 \pm 332.8$ \\
\hline $\mathrm{CON}$ & $1606.5 \pm 540.9$ & $1662.6 \pm 371.0$ \\
\hline \multicolumn{3}{|c|}{ Potassium (mg) } \\
\hline SL & $2896.4 \pm 620.0$ & $3176.0 \pm 934.1$ \\
\hline $\mathrm{CON}$ & $3413.8 \pm 1267.9$ & $3269.9 \pm 680.9$ \\
\hline
\end{tabular}

Table 1 continued

\begin{tabular}{lcc}
\hline & Phase I & Phase II \\
\hline Sodium (mg) & & \\
SL & $5114.4 \pm 2181.0$ & $4908.5 \pm 655.7$ \\
CON & $4933.8 \pm 1064.2$ & $5599.0 \pm 1057.2$ \\
Iron (mg) & & \\
SL & $12.1 \pm 2.7$ & $12.2 \pm 3.8$ \\
CON & $11.7 \pm 5.2$ & $11.1 \pm 2.1$ \\
Zinc $(\mathrm{mg})$ & & \\
SL & $9.1 \pm 3.2$ & $10.0 \pm 2.6$ \\
CON & $10.1 \pm 3.6$ & $10.8 \pm 2.1$ \\
Copper $(\mathrm{mg})$ & & \\
SL & $1.4 \pm 0.6$ & $1.5 \pm 0.2$ \\
CON & $1.5 \pm 0.6$ & $3.8 \pm 1.4$ \\
Manganese $(\mathrm{mg})$ & & $3.6 \pm 0.9$ \\
SL & $3.2 \pm 1.5$ & \\
CON & $3.0 \pm 1.3$ & $53.3 \pm 21.5$ \\
Selenium $(\mu \mathrm{g})$ & & $56.3 \pm 25.8$ \\
SL & $56.7 \pm 17.6$ & \\
CON & $52.9 \pm 17.6$ & \\
\hline
\end{tabular}

Data are mean $\pm \mathrm{SD}$

the risk of infection (Gunzer et al. 2012). Interestingly, in this study, resting immune variables were not significantly modified by the training/nutrition intervention, and the incidence of URTI was not increased in both groups. The number of white blood cells and the incidence of URTI were not significantly altered by the 3 weeks of training, comprising yet nine high intensity training (HIT) sessions and nine low intensity training (LIT) sessions performed after an overnight fast. This result was unexpected considering the high risk of immunodepression classically reported during the early recovery period after HIT sessions, and accentuated by the deprivation in carbohydrates (Gunzer et al. 2012). Only the vitamin D status decreased from pre to post intervention but in similar proportions between groups. This decrease in vitamin $\mathrm{D}$ was accompanied with a slight decrease in $\operatorname{sg}$ A concentration $(d=0.23)$ only in the SL group over weeks of training. This result is in conformity with previous studies reporting a positive correlation between the vitamin D status and the $\operatorname{sIgA}$ secretion $(\mathrm{He}$ et al. 2014). However, it is worth noting that the decrease in vitamin $\mathrm{D}$ could have been also induced by the low direct exposition to UVB, since this study was conducted in the winter season at a high latitude (Paris, $\left.53^{\circ} \mathrm{N}\right)$. While the mean vitamin $\mathrm{D}$ was quite low for all participants, no athletes were deficient in vitamin $\mathrm{D}$ at the end of the protocol. 
Table 2 Weekly evolution of plasma white blood cell counts for SL and CON groups from pre to post phase II

\begin{tabular}{|c|c|c|c|c|c|}
\hline & \multirow[t]{2}{*}{ Pre } & \multicolumn{3}{|l|}{ Phase II } & \multirow[t]{2}{*}{ Post } \\
\hline & & Week 1 & Week 2 & Week 3 & \\
\hline \multicolumn{6}{|c|}{ Leucocytes $\left(/ \mathrm{mm}^{3}\right)$} \\
\hline SL & $4981.8 \pm 1697.5$ & $5000.0 \pm 1649.8$ & $5118.2 \pm 1462.1$ & $4800.0 \pm 1485.5$ & $5230.0 \pm 1682.6$ \\
\hline $\mathrm{CON}$ & $5870.0 \pm 1110.6$ & $5900.0 \pm 829.2$ & $5450.0 \pm 1095.7$ & $5387.5 \pm 800.8$ & $5188.9 \pm 871.0$ \\
\hline \multicolumn{6}{|c|}{ Neutrophils $\left(/ \mathrm{mm}^{3}\right)$} \\
\hline SL & $2695.6 \pm 1442.2$ & $2590.6 \pm 1000.5$ & $2781.1 \pm 1047.9$ & $2541.9 \pm 1127.9$ & $3044.7 \pm 1473.4$ \\
\hline $\mathrm{CON}$ & $3109.2 \pm 1079.2$ & $3211.2 \pm 539.9$ & $2795.8 \pm 867.6$ & $2699.1 \pm 545.4$ & $2512.6 \pm 681.5$ \\
\hline \multicolumn{6}{|c|}{ Eosinophils $\left(/ \mathrm{mm}^{3}\right)$} \\
\hline SL & $111.7 \pm 58.0$ & $101.0 \pm 57.7$ & $107.6 \pm 65.4$ & $94.7 \pm 63.8$ & $111.8 \pm 61.3$ \\
\hline $\mathrm{CON}$ & $172.0 \pm 97.8$ & $172.9 \pm 83.6$ & $166.8 \pm 108.5$ & $170.9 \pm 84.9$ & $150.7 \pm 96.5$ \\
\hline \multicolumn{6}{|c|}{ Basophils $\left(/ \mathrm{mm}^{3}\right)$} \\
\hline SL & $28.5 \pm 14.9$ & $24.7 \pm 17.7$ & $39.8 \pm 16.9$ & $34.7 \pm 16.4$ & $39.6 \pm 24.7$ \\
\hline $\mathrm{CON}$ & $25.3 \pm 9.0$ & $28.4 \pm 5.4$ & $22.6 \pm 13.5$ & $25.9 \pm 12.5$ & $35.7 \pm 28.7$ \\
\hline \multicolumn{6}{|c|}{ Lymphocytes $\left(/ \mathrm{mm}^{3}\right)$} \\
\hline SL & $1736.4 \pm 411.1$ & $1907.5 \pm 826.7$ & $1697.4 \pm 444.3$ & $1737.3 \pm 498.9$ & $1634.2 \pm 456.1$ \\
\hline $\mathrm{CON}$ & $2088.9 \pm 543.5$ & $2011.9 \pm 542.2$ & $1993.5 \pm 480.7$ & $2013.9 \pm 520.1$ & $2013.4 \pm 743.3$ \\
\hline \multicolumn{6}{|c|}{ Monocytes $\left(/ \mathrm{mm}^{3}\right)$} \\
\hline SL & $409.8 \pm 168.9$ & $376.5 \pm 152.0$ & $492.1 \pm 331.1$ & $391.5 \pm 133.4$ & $399.9 \pm 156.0$ \\
\hline $\mathrm{CON}$ & $475.0 \pm 180.0$ & $475.8 \pm 115.1$ & $471.9 \pm 99.2$ & $478.8 \pm 104.7$ & $477.0 \pm 117.6$ \\
\hline
\end{tabular}

Data are mean \pm SD
In our study, the absence of marked perturbations of immunity and URTI despite a chronic physiological stress could be explained by the particularity of our nutrition guidelines. Indeed, participants of the SL group were asked to perform all HIT sessions with high glycogen availability, whereas LIT sessions were performed with low glycogen availability after an overnight fast. However, the total daily carbohydrate intake $\left(5.44 \pm 1.20 \mathrm{~g} \mathrm{~kg}^{-1}\right)$ and energy intake $(2684 \pm 500 \mathrm{kcal})$ were maintained similar to those of the CON group $\left(5.65 \pm 0.99 \mathrm{~g} \mathrm{~kg}^{-1}\right.$ and $\left.2837 \pm 505 \mathrm{kcal}\right)$, likely allowing the maintenance of the immune function throughout the protocol (Marquet et al. 2016). Indeed, carbohydrates are an important source of energy for immune cells (including lymphocytes, neutrophils and macrophages) because their metabolic rates are extremely high (Gunzer et al. 2012). Most importantly all HIT sessions were performed with high glycogen stores, likely inducing a favorable effect on immunity. Costa et al. (2005) reported that training with high $\mathrm{CHO}$ availability lead to a stable glucose level, decreased plasma cortisol level, and an increase in SIgA during 1 week training in well trained triathletes. On the contrary, maintaining a low $\mathrm{CHO}$ diet for the entire week induced a significant increase in cortisol level. In our study, cortisol level was not altered by the nutrition manipulation, probably explaining in large part the maintenance of immune function for all our participants. Indeed, cortisol is known to have a suppressive effects on leukocyte function including immunoglobulin production, lymphocyte proliferation and NK cell activity (Bishop et al. 1999). CHO availability may also increase the anti-inflammatory cytokine response to exercise, as shown by Bishop et al. (2001b) through a significant increase in IL-6 concentration further to a cycling exercise performed in a low $\mathrm{CHO}$ diet $\left(<1 \mathrm{~g} \mathrm{~kg}^{-1}\right.$ per day) for 3 days prior to the exercise. On the contrary, in our study, IL-6 concentration was not significantly modified, probably explained by the $\mathrm{CHO}$ deprivation occurring only during night hours in each day. Finally one other factor which could explain the absence of immunosuppression in the SL group was the intake of a protein snack ( $15 \mathrm{~g}$ protein, $0 \mathrm{~g} \mathrm{CHO}$ ) before going to bed. Initially provided to limit the potential protein catabolism process during the night, ingesting protein in the recovery phase after HIT sessions might have contributed to the maintenance of immune function. Among protein ingested, the specific role of the glutamine amino acid as a privileged energy provider to lymphocytes, macrophages and neutrophils, might be hypothesized (Hiscock and Pedersen 2002). Hence, ingesting a protein shake after exercise might have compensated the fall in plasma glutamine and associated immunosuppression. Moreover, in the SL group the $\mathrm{CHO}$ deprivation period occurred only during night - period during which participants slept and were not exposed to potential pathogen elements and stressors to the immune system such as cold, other people, or mental stress. Finally, well-maintained sleeping patterns throughout the protocol might likely have contributed to the absence of profound 
Table 3 Weekly evolution of salivary IgA and IL-6 concentrations and plasma cortisol concentration for SL and $\mathrm{CON}$ groups from pre to post phase II

\begin{tabular}{|c|c|c|c|c|c|}
\hline & \multirow[t]{2}{*}{ Pre } & \multicolumn{3}{|l|}{ Phase II } & \multirow[t]{2}{*}{ Post } \\
\hline & & Week 1 & Week 2 & Week 3 & \\
\hline \multicolumn{6}{|c|}{$\operatorname{sIgA}(\mu \mathrm{g} / \mathrm{mL})$} \\
\hline SL & $353.7 \pm 188.7$ & $319.1 \pm 167.5$ & $286.7 \pm 154.5$ & $297.4 \pm 132.5$ & $241.6 \pm 98.1^{*}$ \\
\hline $\mathrm{CON}$ & $390.6 \pm 235.0$ & $270.5 \pm 139.7$ & $359.2 \pm 148.4$ & $382.6 \pm 168.5$ & $348.3 \pm 153.9$ \\
\hline \multicolumn{6}{|c|}{ sIL-6 (pg/mL) } \\
\hline SL & $2.6 \pm 0.3$ & $2.6 \pm 0.4$ & $2.7 \pm 0.4$ & $2.6 \pm 0.4$ & $2.6 \pm 0.5$ \\
\hline $\mathrm{CON}$ & $2.8 \pm 0.3$ & $2.8 \pm 0.2$ & $2.6 \pm 0.4$ & $2.7 \pm 0.4$ & $2.7 \pm 0.5$ \\
\hline \multicolumn{6}{|c|}{ Cortisol $(\mu \mathrm{g} / \mathrm{L})$} \\
\hline SL & $157.2 \pm 40.2$ & $140.4 \pm 34.9$ & $159.3 \pm 26.2$ & $155.6 \pm 28.8$ & $148.0 \pm 34.0$ \\
\hline $\mathrm{CON}$ & $177.1 \pm 30.1$ & $180.6 \pm 38.9$ & $169.9 \pm 39.3$ & $176.2 \pm 36.7$ & $155.7 \pm 33.7$ \\
\hline
\end{tabular}

Data are mean \pm SD

* Significantly different from values recorded in pre $(P<0.05)$

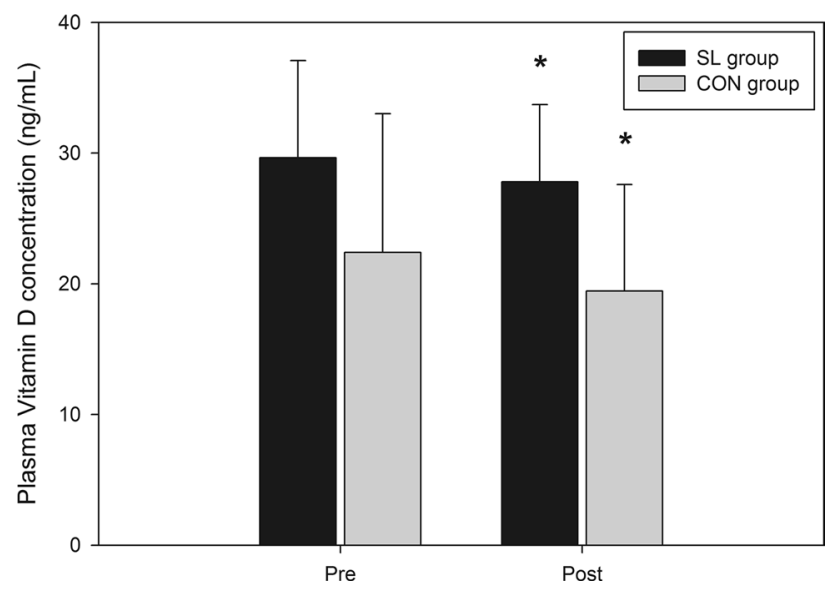

Fig. 3 Evolution of plasma vitamin D concentration for SL and CON groups from pre to post phase II. *Significantly different from pre values $(P<0.05)$

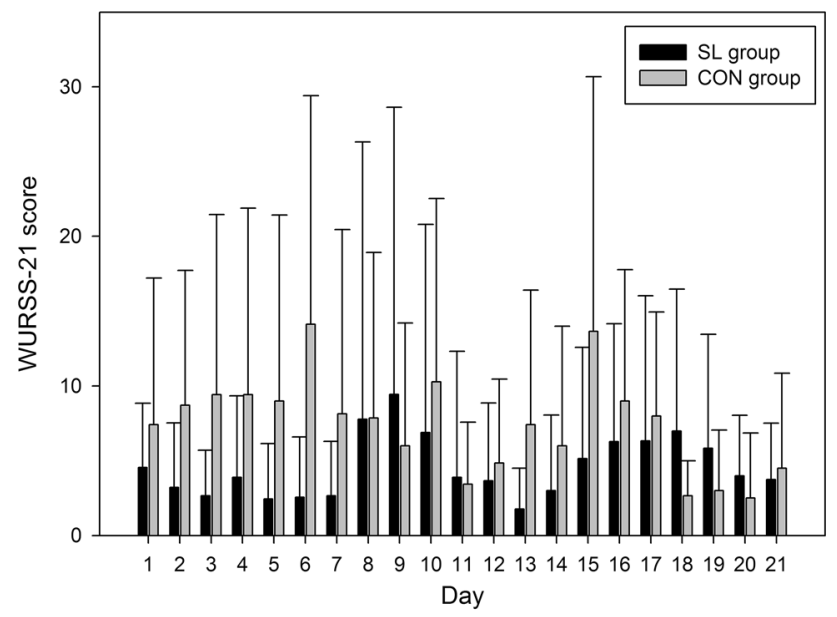

Fig. 4 Daily evolution of the WURSS-21 scores for SL and CON groups during the 21 days of diet and training intervention in phase II perturbations of the immune system and on the very low incidence of URTI.

\section{Effects on sleeping patterns}

Since the first studies having reported that a lack of sleep may significantly alter metabolic, immune and cognitive function, the analyses of sleeping patterns in the sporting context have increased massively (Fullagar et al. 2015). Signs of sleep disturbances (e.g., troubles to fall asleep, increase of wake periods) are often recorded in particular after competitions or high intensity training sessions (Fullagar et al. 2015). For example, Hausswirth et al. (2014) have reported a significant decrease in sleep quality and quantity in triathletes involved in a 3-week overload training program (i.e., $+30 \%$ of habitual training load). In this latter study, the actual sleep duration was decreased by $\sim 30 \mathrm{~min}$ every night during the training program, accompanied by a reduction of sleep efficiency and immobile time. Sleep disruptions are also classically reported in athletic populations training temporarily at high altitude or in hot conditions during training camps (Buchheit et al. 2016; Sargent et al. 2013). Taken together, all data from the literature suggest that intensified training and/or the exposition to physiological stressors (i.e., heat, altitude) increase the risk of sleep disturbance. Within this framework, it can be hypothesized that increasing the physiological stress through a nutritional manipulation during the recovery period could increase and even accentuate the risk of sleep disturbances.

To the best of our knowledge, this study is the first to provide an objective report of sleeping patterns in two groups of trained athletes under different nutrition guidelines (i.e., sleep with low glycogen availability vs. sleep with normal glycogen availability). This experimental situation involved a profound change of dietary habits for all participants, potentially increasing the risk of sleep 
Table 4 Evolution of sleep actigraphy data for SL and CON groups between phase I and phase II

\begin{tabular}{|c|c|c|}
\hline & Phase I & Phase II \\
\hline \multicolumn{3}{|c|}{ Perceived sleep quality (AU) } \\
\hline SL & $3.1 \pm 0.5$ & $3.1 \pm 0.7$ \\
\hline $\mathrm{CON}$ & $3.4 \pm 0.4$ & $3.4 \pm 0.3$ \\
\hline \multicolumn{3}{|c|}{ Time in bed (h:min) } \\
\hline SL & $7: 35 \pm 0: 24$ & $7: 48 \pm 0: 31 *$ \\
\hline $\mathrm{CON}$ & $7: 48 \pm 1: 00$ & $8: 03 \pm 0: 45^{*}$ \\
\hline \multicolumn{3}{|c|}{ Bed time (hh:min) } \\
\hline SL & $23: 53 \pm 0: 37$ & $23: 33 \pm 0: 40 *$ \\
\hline $\mathrm{CON}$ & $23: 46 \pm 1: 08$ & $23: 19 \pm 0: 38^{*}$ \\
\hline \multicolumn{3}{|c|}{ Get-up time (hh:min) } \\
\hline SL & $7: 28 \pm 0: 41$ & $7: 23 \pm 0: 43$ \\
\hline $\mathrm{CON}$ & $7: 45 \pm 0: 59$ & $7: 39 \pm 0: 48$ \\
\hline \multicolumn{3}{|c|}{ Sleep latency (min) } \\
\hline SL & $6 \pm 6$ & $8 \pm 4$ \\
\hline $\mathrm{CON}$ & $10 \pm 7$ & $11 \pm 8$ \\
\hline \multicolumn{3}{|c|}{ Actual sleep time (h:min) } \\
\hline SL & $6: 46 \pm 0: 32$ & $6: 53 \pm 0: 36$ \\
\hline $\mathrm{CON}$ & $6: 30 \pm 0: 50$ & $6: 40 \pm 0: 47$ \\
\hline \multicolumn{3}{|c|}{ Sleep efficiency $(\%)$} \\
\hline SL & $89.1 \pm 3.6$ & $88.2 \pm 3.7^{*}$ \\
\hline $\mathrm{CON}$ & $82.5 \pm 6.8$ & $82.4 \pm 5.9$ \\
\hline \multicolumn{3}{|c|}{ Fragmentation index $(\%)$} \\
\hline SL & $26.6 \pm 6.7$ & $27.9 \pm 8.3$ \\
\hline $\mathrm{CON}$ & $34.1 \pm 11.3$ & $34.2 \pm 8.6$ \\
\hline \multicolumn{3}{|c|}{ Immobile minutes } \\
\hline SL & $392 \pm 31$ & $399 \pm 34$ \\
\hline $\mathrm{CON}$ & $386 \pm 51$ & $396 \pm 44$ \\
\hline
\end{tabular}

Data are mean $\pm \mathrm{SD}$

* Significantly different from values recorded in phase $\mathrm{I}(P<0.05)$

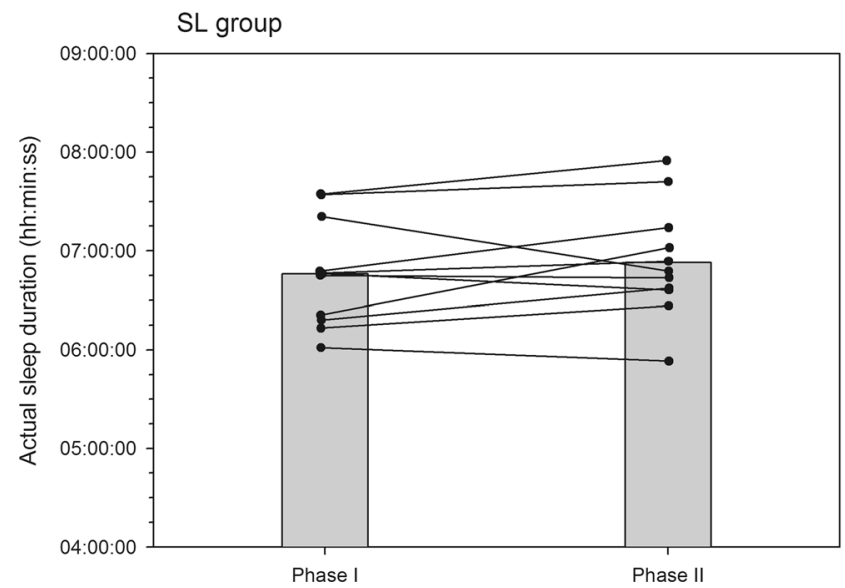

disturbances. Indeed, although carbohydrates are generally recommended at night to facilitate falling asleep (Halson 2014), in our study they were avoided and replaced only by large amounts of vegetables and protein. However, surprisingly sleeping patterns were not markedly modified by the training/nutritional intervention. Sleep latency was not altered and participants even went to bed earlier ( -13 and $-15 \mathrm{~min}, P<0.05$, in the SL and CON group, respectively), while get-up time was not modified. In consequence, actual sleep duration tended to be greater in both groups $(+7$ and $+10 \mathrm{~min}, \mathrm{NS}$, in the SL and CON group, respectively). These results suggest that spontaneously, participants went to bed earlier probably because of the fatigue felt after evening training sessions. The sleep quality was slightly altered since sleep efficiency decreased in the SL group $(-1.1 \%, d=0.25)$ and the fragmentation index tended to increase $(+4.1 \%, P=0.06)$, showing a small increase in wake episodes overnight. However, the overall perceived quality of sleep was not altered throughout the protocol suggesting only minor changes in sleep quality. Altogether, our results suggest that maintaining a normal daily $\mathrm{CHO}$ and calorie intake was effective in maintaining normal sleeping pattern, allowing a good adaptation to training.

\section{Implications for the manipulation of glycogen availability in training programs}

This study has practical implications regarding the withholding of carbohydrates and lowered glycogen availability implemented during short-term endurance training programs: it reveals a minimal effect on immune response and no effects on the occurrence of URTI; it reveals a minimal effect on sleeping patterns in trained triathletes; it raises

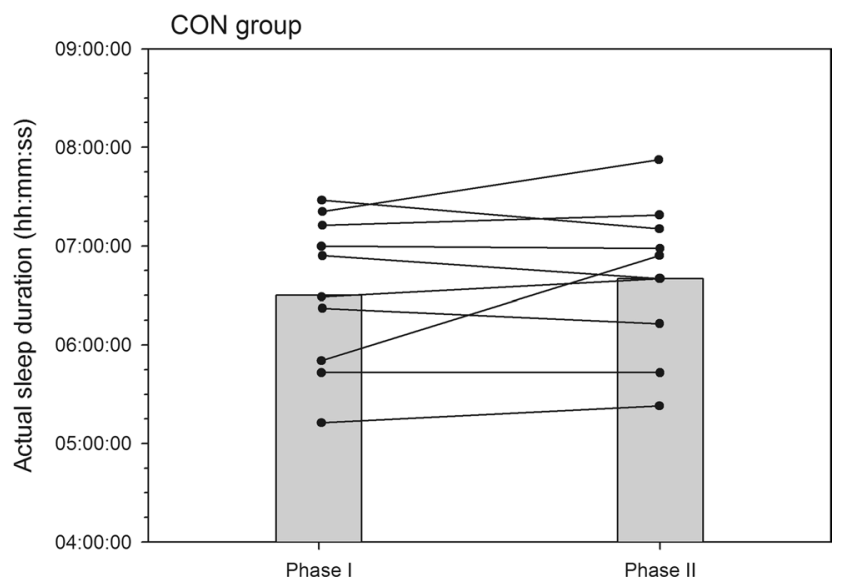

Fig. 5 Mean (shaded bars) and individual cases of actual sleep duration for SL and CON groups during phase I and phase II 

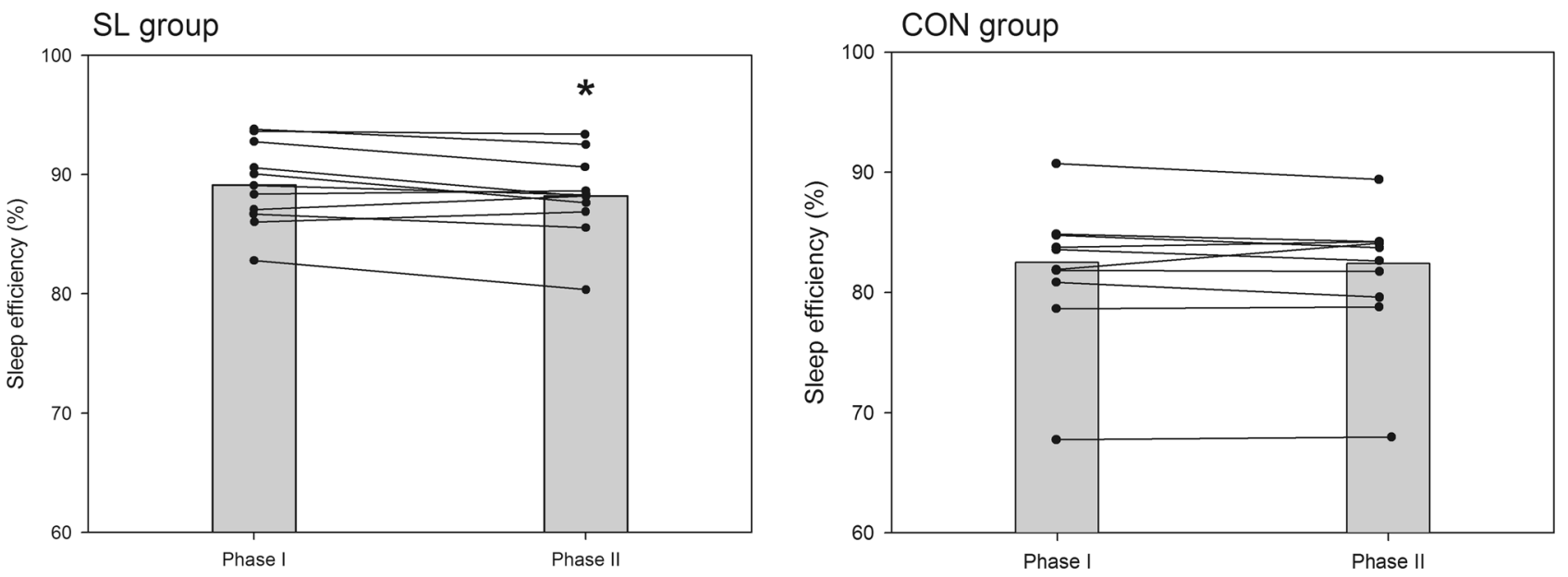

Fig. 6 Mean (shaded bars) and individual cases of sleep efficiency for SL and CON groups during phase I and phase II. *Significantly different from values recorded in phase $\mathrm{I}(P<0.05)$
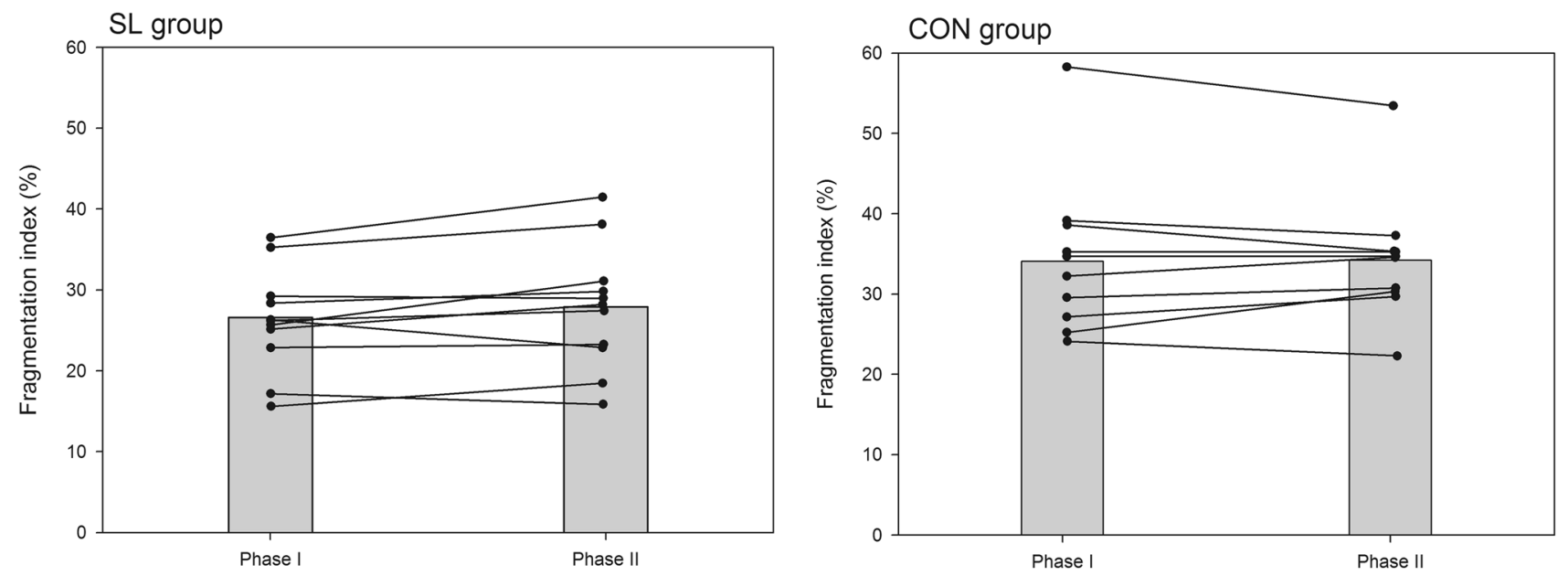

Fig. 7 Mean (shaded bars) and individual cases of fragmentation index for SL and CON groups during phase I and phase II

new insight on the distribution of carbohydrates around training sessions to avoid maladaptation.

Although manipulating carbohydrate availability around training sessions is more and more used by endurance athletes to accentuate the training stimulus and get greater adaptation, to date no study had analyzed the potential side effects on the immune function. As inferred through objective markers of systemic and mucosal immune function as well as subjective markers of infection, the sleep low strategy (3 nights per week over 3 weeks) had no deleterious impact on the immune function. This positive result is likely explained by the maintenance of normal daily $\mathrm{CHO}$ (around $6 \mathrm{~g} \mathrm{~kg}^{-1} \mathrm{day}^{-1}$ ) and energy intake (i.e., the $\mathrm{CHO}$ intake was matched between groups in this study). However, additional studies are necessary to confirm this result and analyze the impact of various dietary/ training manipulations on the immune function. Moreover, in our study, the vitamin D status decreased in both groups accompanied with a slight reduction in the $\operatorname{sgA}$ secretion in the SL group $(d=0.23)$, suggesting a slight reduction in immune defense. Accordingly, a preliminary vitamin D status assessment possibly followed by a dietary supplementation in vitamin D should be considered to optimize the immune status before engaging in a sleep low program, especially if started in the winter season.

Sleeping is often considered by coaches and trainers as the most effective recovery strategy. However, very limited data is available on the potential factors of influence, such as dietary feedings according to the distribution of training sessions. This study shows for the first time that sleeping patterns of trained athletes were not altered by a sleep low training strategy. Maintaining a normal $\mathrm{CHO}$ and energy 
intake every day of the training program would be enough to counterbalance the potential negative impact of withholding $\mathrm{CHO}$ in the recovery phase after exercise before going to bed.

This study provides new evidence on the optimization of the carbohydrate distribution over day to limit the risk of maladaptation to training. High intensity training sessions should be performed with high glycogen availability to maintain blood glucose concentration and thus immune function. The deprivation in $\mathrm{CHO}$ should occur in the recovery phase after HIT sessions organized overnight, to facilitate the glycogen depletion. Indeed, the night can be considered as a suitable period to withhold $\mathrm{CHO}$ (more convenient and easiest to endure a 7-8 h period of low glycogen availability). Ingesting a dinner rich in vitamin, minerals (mainly from vegetables) and proteins might reinforce the immune defenses. Exercises with low glycogen availability should be performed at a low intensity (fasted in the morning) to lower the risk of infection. Finally, a particular attention should be brought on the ingestion of sufficient amounts of $\mathrm{CHO}$ between morning and evening sessions (from breakfast to afternoon snack) to provide a normal amount of $\mathrm{CHO}$ (around $6 \mathrm{~g} \mathrm{~kg}^{-1} \mathrm{day}^{-1}$ in this study). The use of liquid sources of $\mathrm{CHO}$ like sports drinks and gels can facilitate the ingestion of enough $\mathrm{CHO}$ in a short period of time.

\section{Conclusion}

The purpose of this study was to test the effects of a 3-week sleep low training strategy (involving a withholding of $\mathrm{CHO}$ overnight) on immune function, the incidence or URTI and sleep patterns in trained endurance athletes. While the CON group maintained a normal $\mathrm{CHO}$ availability all time during the protocol, the SL group stopped $\mathrm{CHO}$ intake from the afternoon training session until the next morning training session, so that they spend all night with reduced glycogen availability. The main findings were that: (i) markers of systemic and mucosal immunity and the incidence of URTI were not significantly modified by the dietary intervention; (ii) sleep efficiency slightly decreased and the fragmentation index tended to increase only in the SL group. Overall, results derived from this study suggest that the risk of maladaptation to training is minimal when withholding $\mathrm{CHO}$ overnight between high and low intensity training sessions, in the condition of maintaining a normal daily $\mathrm{CHO}$ and energy intake concentrated earlier over day.

Acknowledgments The authors wish to acknowledge the French Institute of Sport (INSEP) for supporting the study and Gatorade France for providing sports drinks used during the training program and performance tests described in this article.

\section{Compliance with ethical standards}

Conflict of interest The authors report no conflict of interest.

Open Access This article is distributed under the terms of the Creative Commons Attribution 4.0 International License (http://creativecommons.org/licenses/by/4.0/), which permits unrestricted use, distribution, and reproduction in any medium, provided you give appropriate credit to the original author(s) and the source, provide a link to the Creative Commons license, and indicate if changes were made.

\section{References}

Afaghi A, O'Connor H, Chow CM (2008) Acute effects of the very low carbohydrate diet on sleep indices. Nutr Neurosci 11:146154. doi:10.1179/147683008X301540

Barrett B, Brown RL, Mundt MP, Thomas GR, Barlow SK, Highstrom AD, Bahrainian M (2009) Validation of a short form Wisconsin Upper Respiratory Symptom Survey (WURSS-21). Health Qual Life Outcomes 7:76. doi:10.1186/1477-7525-7-76

Bartlett JD, Hawley JA, Morton JP (2015) Carbohydrate availability and exercise training adaptation: too much of a good thing? Eur J Sport Sci 15:3-12. doi:10.1080/17461391.2014.920926

Bermon S (2007) Airway inflammation and upper respiratory tract infection in athletes: is there a link? Exerc Immunol Rev 13:6-14

Bishop NC, Blannin AK, Walsh NP, Robson PJ, Gleeson M (1999) Nutritional aspects of immunosuppression in athletes. Sports Med 28:151-176

Bishop NC, Walsh NP, Haines DL, Richards EE, Gleeson M (2001a) Pre-exercise carbohydrate status and immune responses to prolonged cycling: I. Effect on neutrophil degranulation. Int J Sport Nutr Exerc Metab 11:490-502

Bishop NC, Walsh NP, Haines DL, Richards EE, Gleeson M (2001b) Pre-exercise carbohydrate status and immune responses to prolonged cycling: II. Effect on plasma cytokine concentration. Int J Sport Nutr Exerc Metab 11:503-512

Buchheit M, Cholley Y, Lambert P (2016) Psychometric and physiological responses to a preseason competitive camp in the heat with a 6-hour time difference in elite soccer players. Int J Sports Physiol Perform 11:176-181. doi:10.1123/ijspp.2015-0135

Costa RJ, Jones GE, Lamb KL, Coleman R, Williams JH (2005) The effects of a high carbohydrate diet on cortisol and salivary immunoglobulin A (s-IgA) during a period of increase exercise workload amongst Olympic and Ironman triathletes. Int J Sports Med 26:880-885. doi:10.1055/s-2005-837467

Fahlman MM, Engels HJ (2005) Mucosal IgA and URTI in American college football players: a year longitudinal study. Med Sci Sports Exerc 37:374-380

Fietze I, Strauch J, Holzhausen M, Glos M, Theobald C, Lehnkering H, Penzel T (2009) Sleep quality in professional ballet dancers. Chronobiol Int 26:1249-1262. doi:10.3109/07420520903221319

Fullagar HH, Skorski S, Duffield R, Hammes D, Coutts AJ, Meyer $T$ (2015) Sleep and athletic performance: the effects of sleep loss on exercise performance, and physiological and cognitive responses to exercise. Sports Med 45:161-186. doi:10.1007/ s40279-014-0260-0

Gleeson M (2007) Immune function in sport and exercise. J Appl Physiol 103:693-699. doi:10.1152/japplphysiol.00008.2007

Gleeson M, McDonald WA, Pyne DB, Cripps AW, Francis JL, Fricker PA, Clancy RL (1999) Salivary IgA levels and infection risk in elite swimmers. Med Sci Sports Exerc 31:67-73 
Gunzer W, Konrad M, Pail E (2012) Exercise-induced immunodepression in endurance athletes and nutritional intervention with carbohydrate, protein and fat-what is possible, what is not? Nutrients 4:1187-1212. doi:10.3390/nu4091187

Halson SL (2014) Sleep in elite athletes and nutritional interventions to enhance sleep. Sports Med 44(Suppl 1):S13-S23. doi:10.1007/s40279-014-0147-0

Hansen AK, Fischer CP, Plomgaard P, Andersen JL, Saltin B, Pedersen BK (2005) Skeletal muscle adaptation: training twice every second day vs. training once daily. J Appl Physiol 98:93-99. doi:10.1152/japplphysiol.00163.2004

Hausswirth C, Louis J, Aubry A, Bonnet G, Duffield R, Le Meur Y (2014) Evidence of disturbed sleep and increased illness in overreached endurance athletes. Med Sci Sports Exerc 46:10361045. doi:10.1249/MSS.0000000000000177

He CS, Fraser WD, Gleeson M (2014) Influence of vitamin d metabolites on plasma cytokine concentrations in endurance sport athletes and on multiantigen stimulated cytokine production by whole blood and peripheral blood mononuclear cell cultures. ISRN Nutr 2014:820524. doi:10.1155/2014/820524

Hiscock N, Pedersen BK (2002) Exercise-induced immunodepression-plasma glutamine is not the link. J Appl Physiol 93:813822. doi:10.1152/japplphysiol.00048.2002

Hoffman-Goetz L, Pedersen BK (1994) Exercise and the immune system: a model of the stress response? Immunol Today 15:382387. doi:10.1016/0167-5699(94)90177-5

Hooper SL, Mackinnon LT, Howard A, Gordon RD, Bachmann AW (1995) Markers for monitoring overtraining and recovery. Med Sci Sports Exerc 27:106-112

Impey SG et al (2016) Fuel for the work required: a practical approach to amalgamating train-low paradigms for endurance athletes. Physiol Rep. doi:10.14814/phy2.12803

Kushida CA, Chang A, Gadkary C, Guilleminault C, Carrillo O, Dement WC (2001) Comparison of actigraphic, polysomnographic, and subjective assessment of sleep parameters in sleepdisordered patients. Sleep Med 2:389-396

Lane SC et al (2015) Effects of sleeping with reduced carbohydrate availability on acute training responses. J Appl Physiol 119:643655. doi:10.1152/japplphysiol.00857.2014

Leeder J, Glaister M, Pizzoferro K, Dawson J, Pedlar C (2012) Sleep duration and quality in elite athletes measured using wristwatch actigraphy. J Sports Sci 30:541-545. doi:10.1080/02640414.201 2.660188

Lindseth G, Lindseth P, Thompson M (2013) Nutritional effects on sleep. West J Nurs Res 35:497-513. doi:10.1177/0193945911416379

Marquet LA, Brisswalter J, Louis J, Tiollier E, Burke LM, Hawley JA, Hausswirth C (2016) Enhanced endurance performance by periodization of $\mathrm{CHO}$ intake: "sleep low" strategy. Med Sci Sports Exerc. doi:10.1249/MSS.0000000000000823

Matthews CE, Ockene IS, Freedson PS, Rosal MC, Merriam PA, Hebert JR (2002) Moderate to vigorous physical activity and risk of upper-respiratory tract infection. Med Sci Sports Exerc 34:1242-1248

Mitchell JB, Pizza FX, Paquet A, Davis BJ, Forrest MB, Braun WA (1998) Influence of carbohydrate status on immune responses before and after endurance exercise. J Appl Physiol 84:1917-1925

Morton JP et al (2009) Reduced carbohydrate availability does not modulate training-induced heat shock protein adaptations but does upregulate oxidative enzyme activity in human skeletal muscle. J Appl Physiol 106:1513-1521. doi:10.1152/ japplphysiol.00003.2009

Myllymaki T et al (2011) Effects of vigorous late-night exercise on sleep quality and cardiac autonomic activity. J Sleep Res 20:146-153. doi:10.1111/j.1365-2869.2010.00874.x

Nieman DC (1998) Influence of carbohydrate on the immune response to intensive, prolonged exercise. Exerc Immunol Rev 4:64-76

Nieman DC (2000) Is infection risk linked to exercise workload? Med Sci Sports Exerc 32:S406-S411

Nieman DC, Johanssen LM, Lee JW, Arabatzis K (1990) Infectious episodes in runners before and after the Los Angeles Marathon. J Sports Med Phys Fitness 30:316-328

Nieman DC, Henson DA, Austin MD, Sha W (2011) Upper respiratory tract infection is reduced in physically fit and active adults. Br J Sports Med 45:987-992. doi:10.1136/bjsm.2010.077875

Pyne DB, Baker MS, Fricker PA, McDonald WA, Telford RD, Weidemann MJ (1995) Effects of an intensive 12-wk training program by elite swimmers on neutrophil oxidative activity. Med Sci Sports Exerc 27:536-542

Sadeh A (2011) The role and validity of actigraphy in sleep medicine: an update. Sleep Med Rev 15:259-267. doi:10.1016/j. smrv.2010.10.001

Samuels C (2008) Sleep, recovery, and performance: the new frontier in high-performance athletics. Neurol Clin 26:169-180, ix-x. doi:10.1016/j.ncl.2007.11.012

Sargent C et al (2013) The impact of altitude on the sleep of young elite soccer players (ISA3600). Br J Sports Med. 47(Suppl 1):i86-i92. doi:10.1136/bjsports-2013-092829

Shepard RJ, Shek PN (1996) Impact of physical activity and sport on the immune system. Rev Environ Health 11:133-147

Taylor SR, Rogers GG, Driver HS (1997) Effects of training volume on sleep, psychological, and selected physiological profiles of elite female swimmers. Med Sci Sports Exerc 29:688-693

Van Proeyen K, Szlufcik K, Nielens H, Ramaekers M, Hespel P (2011) Beneficial metabolic adaptations due to endurance exercise training in the fasted state. J Appl Physiol 110:236-245. doi:10.1152/japplphysiol.00907.2010

Yeo WK, Paton CD, Garnham AP, Burke LM, Carey AL, Hawley JA (2008) Skeletal muscle adaptation and performance responses to once a day versus twice every second day endurance training regimens. J Appl Physiol 105:1462-1470. doi:10.1152/ japplphysiol.90882.2008 